\title{
AKTIONSFORSCHUNG UND IHRE BEITRÄGE ZUR WISSENSCHAFTLICHE METHODE: ALLGEMEINE ASPEKTE
}

\section{ORIGINALER ARTIKEL}

DENDASCK, Carla Viana ${ }^{1}$

DENDASCK, Carla Viana. Aktionsforschung und ihre beiträge zur wissenschaftliche methode: allgemeine aspekte. Revista Científica Multidisciplinar Núcleo do Conhecimento. Jahr. 06, Hrsg. 11, Vol. 11, S. 118-135. Oktober 2021. ISSN: 2448-0959, Zugangslink: https://www.nucleodoconhecimento.com.br/bildungde/wissenschaftliche-methode, DOI: 10.32749/nucleodoconhecimento.com.br/bildung-de/wissenschaftliche-methode

\section{ZUSAMMENFASSUNG}

Aufgrund der zunehmenden Popularität und der Möglichkeit, Aktionsforschung zu nutzen, begann das Instrument signifikant verwendet zu werden, aber mit einem noch nicht konsolidierten Konzept wurde der Be bmgriff auf abstrakte Weise angewendet, ohne tiefe und detaillierte Interpretationen der Verwendung in verschiedenen Kontexten. Es soll in diesem Artikel die Bedeutung des Begriffs verdeutlichen und wie diese Art von Instrument am besten angewendet werden kann, indem es im Bereich der wissenschaftlich-methodischen Strenge handelt. Das Forschungsproblem lautet: Welche Möglichkeiten gibt es, Die Aktionsforschung, ihre Stadien und Grundlagenpflege zu nutzen? Die Relevanz des Tools liegt darin, dass es die Durchführung einer Forschung systematisiert, kontinuierlich und empirisch basiert ermöglicht. So wird über die Rolle der Theorie in der Aktionsforschung diskutiert und die Merkmale ihrer grundlegenden Phasen aufgezeigt. Einige häufige Fragen im Zusammenhang mit der Methode, wie die Beteiligung des Forschers, die soziale

\footnotetext{
${ }^{1}$ Theologe, PhD in Clinical Psychoanalysis. Er arbeitet seit 15 Jahren mit Scientific Methodology (Research Method) in der wissenschaftlichen Produktionsberatung von Master- und Doktoranden. Spezialist für Marktforschung und Forschung mit Schwerpunkt Gesundheit. Doktorand in Kommunikation und Semiotik (PUC $\mathrm{SP})$.

RC: 102352
}

Zugangslink: https://www.nucleodoconhecimento.com.br/bildung-de/wissenschaftlichemethode 
Funktion der Reflexion, die Notwendigkeit, das erworbene Wissen und die Ethik in der Forschung zu verwalten, sollten berücksichtigt werden. Schließlich werden einige Aktionsforschungs-"Modelle" vorgestellt, die den Forschern bei der methodischen Auswahl und Organisation helfen können.

Schlüsselwörter: Aktionssuche, Suchmethoden, Suchwerkzeuge.

\section{EINLEITUNG}

Aktionsforschung ist eine Art von Forschung, die vom Teilnehmer eine engagierte Haltung verlangt (TRIPP, 2005). Es handelt sich um eine Strategie, die in gewisser Weise im Gegensatz zur traditionellen Forschung steht, die als "unabhängig", "nicht reaktiv" und "objektiv" angesehen wird (ENGEL, 2000). Wie der Begriff selbst andeutet, zielt die Aktionsforschung darauf ab, Forschung mit Aktion, dh Praxis, zu verbinden. So werden Wissen und Verständnis als Teil der Praxis verstanden und sind dann eine Möglichkeit, Forschung in Situationen einzusetzen, in denen der Forscher auch eine praktische Neigung hat und das Verständnis einer Theorie verbessern möchte (KOERICH et al., 2009). Die Argumente, die der Aktionsforschung entgegenstehen, sind insbesondere auf die Tatsache zurückzuführen, dass der Forscher auf dem untersuchten Gebiet tätig ist, was im Einklang mit den ethischen Annahmen in der wissenschaftlichen Forschung stünde, die die Befreiung des Forschers als Teil des Prozesses der wissenschaftlichen Qualität empfehlen, wobei darauf hingewiesen wird, dass diese Ausnahme dafür verantwortlich wäre, Klarheit in die Argumente des Forschers zu bringen, der von der Tendenz zur Erstellung von Ergebnissen abwesend wäre (TRIPP, 2005; KOERICH et al., 2009).

Diese Art der Forschung entstand aus der Notwendigkeit, eine bestehende Lücke zwischen den Achsen von Theorie und Praxis zu überwinden. Eines seiner vorherrschenden Merkmale ist, dass es sich um eine Forschung handelt, von der aus es versucht, auf innovative Weise in die Praxis einzugreifen, während der Entwicklung der Forschung. Es ist nicht nur eine Empfehlung, die in der Endphase erscheint (ENGEL, 2000). Dieser Aspekt wurde insbesondere von Programmen untersucht, die darauf abzielen, Pragmatismus als Endprodukt $z u$ fördern, wie in den RC: 102352

Zugangslink: https://www.nucleodoconhecimento.com.br/bildung-de/wissenschaftlichemethode 
Graduiertenprogrammen für Ingenieurwesen, Wirtschaft oder sogar in den professionellen Master- und Doktoratsprogrammen.

Einer der ersten, der Die Aktionsforschung in den akademischen Kontext einführte, war der deutsche Psychologe Kurt Lewin. Doch bereits in den 1960er Jahren eignete sich die Soziologie das Konzept schnell an (TRIPP, 2005). Es wurde davon ausgegangen, dass der Forscher diese passivere, isoliertere Haltung aufgeben und damit die Konsequenzen übernehmen sollte, die durch die Ergebnisse seiner Forschung verursacht wurden, und dass solche Ergebnisse in die Praxis umgesetzt werden sollten, um so einer Nachfrage gerecht zu werden, die mehr Beitrag aus dem akademischen Kontext zur praktischen Welt suchte (FRANCO, 2005).

Diese Suche entsteht dann im Wechsel der theoretischen Haltung zu einer praktischen Haltung, die in den Lauf der Ereignisse eingreifen kann. In diesem Zusammenhang sind neben seiner Anwendbarkeit in den Sozialwissenschaften und der Psychologie selbst seine Anwendungsmöglichkeiten heute breit gefächert, was die Relevanz dieser Studie rechtfertigt. Eine seiner neuen Möglichkeiten ist der Einsatz im Bildungsbereich, der als Antwort auf die Anforderungen entwickelt wurde, die mit der Umsetzung der Bildungstheorie in der Unterrichtspraxis, d.h. im Unterricht, verbunden sind (ENGEL, 2000). Heute kann eines der Ergebnisse der Aktionsforschung im Universum der Bildung durch die zahlreichen Materialien gefunden werden, die mit aktiven Methoden arbeiten, sogar steigern, so dass das Konzept der aktiven Methodik von einigen Forschern als Disziplin und unabhängige Methodik integriert wird.

Wie bereits erwähnt, wurden Theorie und Praxis nicht als inhärente Bestandteile des Berufslebens wahrgenommen. Angesichts solcher Veränderungen begann die Umsetzung der Aktionsforschung in den unterschiedlichsten Kontexten (TRIPP, 2005). Ziel ist es, Forschern und Fachleuten zu helfen, die Probleme zu lösen, die ihre tägliche Arbeitspraxis betreffen, indem sie sich viel stärker in die Forschung einbringen (MIRANDA; RESENDE, 2006). Damit wurden im Bildungsbereich Werte, Wahrnehmungen und Überzeugungen in der Ausübung des Lehrens-Lernens empirisch evaluiert (ENGEL, 2000). Es versteht sich, dass diese Art von Forschung 
von den unterschiedlichsten Akademikern mit guten Augen betrachtet wurde. Es ist eine attraktive Forschung, weil sie den Forscher zu einem spezifischen und unmittelbaren Ergebnis in Bezug auf ein bestimmtes Forschungsproblem (GRITTEM; MEIER; ZAGONEL, 2008).

Es wird erwähnt, dass sich die Aktionsforschung als effizientes Instrument für die berufliche Entwicklung mehrerer Forscher erwiesen hat. Es wird darauf hingewiesen, dass dieser Prozess von "innen nach außen" stattfindet, da ein Teil der Anliegen und Interessen der an der Forschungspraxis beteiligten Personen (TRIPP, 2005). Die Probanden beschäftigen sich mit Themen, die die eigene berufliche Entwicklung und auch die persönliche Entwicklung fördern. Der entgegengesetzte Ansatz der Aktionsforschung ist gerade traditionelle Forschung, denn Forschung findet von "out into in" statt (ENGEL, 2000). In diesem kanonischen Prozess füttert der Forscher seine Studie mit den Wahrnehmungen, die aus einer externen Erfahrung stammen, und diese Erfahrung wird durch bestimmte Forschungsinstrumente (wie Fragebögen und Interviews) gesammelt (FRANCO, 2005). Es ist jedoch nicht beabsichtigt, einen idealen Ansatz zu diskutieren, da es sich um einzigartige und unterschiedliche Vorschläge handelt. Dies sind zwei Möglichkeiten, die Natur der wissenschaftlichen Forschung zu betrachten.

Es wird angenommen, dass wissenschaftliche Wahrheiten in der Welt außerhalb der Forschung existieren, und es liegt am Wissenschaftler, solche Wahrheiten zu entdecken, einschließlich der Fähigkeit, einzugreifen, um solche Wahrheiten zu testen. In der wissenschaftlichen Forschung lässt sich jedoch ableiten, dass es Möglichkeiten gibt, das Wesen einer Forschung zu betrachten, so dass es keine absoluten wissenschaftlichen Wahrheiten gibt, weil alles Wissen provisorisch ist und sich je nach dem historischen Kontext, in dem man lebt, ändert (MIRANDA; RESENDE, 2006).

Phänomene werden sowohl beobachtet als auch entsprechend solcher historischen und kulturellen Besonderheiten interpretiert. Es wird auch erwähnt, dass die Forschungsmuster selbst Veränderungen unterliegen, weil sich die Wissenschaft jeden Tag weiterentwickelt. Es gibt keine universelle und historische wissenschaftliche 
Methodik, sondern unterschiedliche Sichtweisen auf die Welt unter wissenschaftlicher Voreingenommenheit (GRITTEM; MEIER; ZAGONEL, 2008). Die Aktionsforschung nähert sich in diesem Sinne einer praktischeren Sicht auf alltägliche Phänomene und Situationen. Somit werden wissenschaftliche Erkenntnisse als vorläufig und kontextabhängig angesehen.

Unter Berücksichtigung des Bildungsbereichs versteht es sich, dass Lehrer nicht nur Konsumenten einer von anderen durchgeführten Forschung sind, sondern ihre eigene Unterrichtspraxis durch die Ergebnisse dieser Studie transformieren sollten (ENGEL, 2000). So wird Die Aktionsforschung als ideales Instrument für die Durchführung einer Forschung konzipiert, die Theorie mit Praxis verbinden will. In diesem Sinne besteht das allgemeine Ziel dieser Forschung darin, durch eine explorative und deskriptive Studie über die Anwendungsmöglichkeiten der Aktionsforschung in jedem Umfeld sozialer Interaktion nachzudenken. Das Forschungsproblem lautet: Welche Möglichkeiten gibt es, Aktionsforschung und ihre grundlegenden Schritte und Pflege zu nutzen? Basierend auf dem Problem zielt es darauf ab, zu überprüfen, wie eine Umgebung, in der soziale Interaktion stattfindet und die ein reales und dringendes Problem hat, von dieser Art von Studie profitieren kann, einer Umgebung, die Menschen, Aufgaben und Verfahren umfasst, die Hilfe benötigen.

\section{AKTIONSFORSCHUNG UND IHR EINFLUSS AUF DIE SOZIALE PRAXIS}

Die Literatur beschreibt den Verlauf der Aktionsforschung seit den 1940er Jahren. Aus einer breiten Sicht kann dies in zwei bedeutende Perioden eingeordnet werden: Die erste ist mit einer amerikanischen Strömung verbunden, die durch die Entstehung des von Kurt Lewis in der Zeit vor dem Zweiten Weltkrieg geprägten Begriffs gekennzeichnet ist (MIRANDA; RESENDE, 2006). Diese Phase dauert bis in die 1960er Jahre. Der zweite Moment wiederum ist geprägt von einer europäischen Strömung, die den Zeitraum von den 1960er Jahren bis heute abdeckt (BARBIER, 2002; MORIN, 2004). Die beiden Stränge geben einen Überblick über diese Art von Forschung und konzentrieren sich dabei auf den qualitativen Ansatz der RC: 102352

Zugangslink: https://www.nucleodoconhecimento.com.br/bildung-de/wissenschaftlichemethode 
sozialwissenschaftlichen Forschung. Die Konzepte, Begründungen und methodischen Erklärungen, die durch theoretische und methodische Verbindungen konstruiert wurden, grenzten die Forschung ab, die als die Forschung bezeichnet wurde (BARBIER, 2002; MORIN, 2004).

Daraus ergibt sich der Begriff der Intervention, und dieser kann von einer Position, die eher mit experimentellen Studien verbunden ist, bis hin zu sozialen Aktionsprojekten, deren Zweck die Lösung der verschiedensten sozialen Probleme ist, variieren (THIOLLENT, 1984). Aufgrund der verschiedenen Konzepte und Autoren, die begannen, Die Aktionsforschung zu diskutieren, wurden mehrere Lesarten und Interpretationen konstant und tendierten nun zu einer erklärenderen (experimentellen) Perspektive oder zu einer umfassenderen (phänomenologischen oder dialektischen) Perspektive (TRIPP, 2005). Die Aktionsforschung entpuppt sich zunächst als Kritik am Positivismus, auch wenn man versteht, dass sich nicht alle von einem solchen Verständnis entfernt haben. Prinzipiell werden sie auch als Ansätze umfassender Natur gesehen, weil sie die soziale Realität als etwas anerkennen, das nicht existiert oder als unabhängig und autonom anerkannt werden kann (FRANCO, 2005). Es ist also eine subjektive Realität und als solche wird es durch individuelle Handlungen konstruiert und aufrechterhalten.

Es sind diese einzelnen Handlungen, die dieser Realität im Konstruktionsprozess Bedeutungen und Bedeutungen zuschreiben (CARR; KEMMIS, 1988). Neben anderen Aspekten kann auch erwähnt werden, dass es eine Parallele zwischen diesen neuen Neuinterpretationen der Aktionsforschung gibt. Die französischen bzw. kanadischen Ansätze, die von René Barbier (2002) bzw. André Morin (2004) vorgeschlagen wurden, verbinden. Indem man diese Autoren sowie einige ihrer Werke als Referenz nimmt, ist es nicht beabsichtigt, ihr Denken und ihre Form der Produktion dieser Art von Studie zu verallgemeinern, sondern vielmehr einige ihrer allgemeinen Elemente aufzulisten, denn diese ermöglichen es uns, die Entfaltung der Aktionsforschung in den unterschiedlichsten Kontexten auf tiefgreifende Weise zu verstehen (TRIPP, 2005). Der Bereich der Bildung ist, wie bereits erwähnt, eine der Möglichkeiten von Räumen, in denen Die Aktionsforschung maßgeblich wirken kann. Darüber hinaus wird RC: 102352

Zugangslink: https://www.nucleodoconhecimento.com.br/bildung-de/wissenschaftlichemethode 
der Aktionsforschung eine Bedeutung zugeschrieben: Sie wird als epistemologische Revolution eingestuft (BARBIER, 2002).

Es wird so genannt, weil der Vorschlag bis dahin auf dem Gebiet der Humanwissenschaften nicht wesentlich erforscht worden war. Daher verstand man, dass Aktionsforschung eine Art "Kunst der klinischen Strenge" war (BARBIER, 2002). Daher kann es mit einer Kollektivität entwickelt werden. Das Ziel ist es also, einen Teil dieser Gesellschaft auf eine weniger entfernte, weniger objektive Weise zu analysieren, wie es in der traditionellen Forschung der Fall ist, in der Neutralität und Distanzierung vom Forscher verteidigt werden (TRIPP, 2005). Damit diese Art von Studie jedoch durchführbar wird, impliziert dies eine Änderung der Haltung des Subjekts, sei es ein Individuum oder eine soziale Gruppe, in Bezug auf ihre Realität (KOERICH et al., 2009). Es ist dann notwendig, reale Wahrnehmungen und Meinungen über ein bestimmtes Phänomen zu liefern, das im Alltag erlebt wird. Der Forscher erfasst also dieses Phänomen und hält es fest, indem er diese Wahrnehmungen, Werte und Überzeugungen des analysierten Subjekts/der analysierten Gruppe mit ihren theoretischen Vorstellungen vereint.

Die Ausübung von Aktionsforschung impliziert, dass der Forscher eine offene systemische Sicht hat, um die beobachteten Phänomene aufzuzeichnen. Daher muss es zum Zeitpunkt der Registrierung bestimmte Prozesse wie Organisation, Information, Wahrnehmungen, Werte, Überzeugungen mit den Quellen kombinieren, aus denen es seine Studie gemäß der erforderlichen wissenschaftlichen Strenge aufbaut (MIRANDA; RESENDE, 2006). Die Nähe des Forschers des untersuchten Phänomens ist einer der Aspekte, die die Aktionsforschung von den eher kanonischen, theoretischen, entfernt. Dies ist auf genau die Annahmen zurückzuführen, die diesem Tool seit seiner Entstehung zugrunde liegen (ENGEL, 2000). Die Pionierautoren auf diesem Gebiet kritisieren in ihren Studien die Wirkung des Positivismus auf dem Gebiet der Sozialwissenschaften, da dieser Trend der Forschungstätigkeit Grenzen setzte und dies auf einigen Säulen wie Objektivität, Rationalität und Wahrheit beruhte (KOERICH et al., 2009). 
Die Aktionsforschung ist mit der Idee des ständigen Wandels verbunden und basiert daher auf der Annahme, dass sich die Akteure, die die Realität ausmachen, auch in einem ständigen Transformationsprozess befinden (TRIPP, 2005). Angesichts dieser Mentalität entstand die Notwendigkeit, über den Begriff der Forschung zusammen mit dem Handeln nachzudenken, das heißt, zu üben, und damit begannen wir, über die Strategien zur Lösung sozialer Probleme aus einem anderen Ansatz nachzudenken (FRANCO, 2005). In diesem Zusammenhang bringen Studien, die diesen Forschungsansatz wählen, die Bereiche der Praxis, des sozialen Handelns, mit dem theoretischen, erkenntnistheoretischen in Einklang. Die Praxis, d.h. die Handlung, betrifft alles, was in einem gegebenen Kontext konstituiert und aufrechterhalten wird und umfasst damit auch die Handlung und tägliche Erfahrung eines Subjekts (GRITTEM; MEIER; ZAGONEL, 2008). Aktionsforschung ist mehr als ein methodischer Ansatz. Dies ist eine Position angesichts wesentlicher erkenntnistheoretischer Fragen, wie dem Verhältnis von Subjekt und Objekt, Theorie und Praxis.

\section{MERKMALE EINER AKTIONSFORSCHUNG}

Die Aktionsforschung umfasst einen empirischen Prozess, der die Identifizierung des Problems in einem sozialen und/oder institutionellen Kontext, die Erhebung von Daten über das betreffende Problem und die Analyse der Bedeutungen und Bedeutungen, die den Daten der Teilnehmer zugeschrieben werden, ermöglicht (TRIPP, 2005). Neben der Identifizierung von Veränderungsbedarf und der Erhebung möglicher notwendiger Lösungen greift die Aktionsforschung auch in das praktische Umfeld ein, da sie Transformation provoziert und vorantreibt (ENGEL, 2000). Es ist ein methodisches Werkzeug, das in der Lage ist, Theorie mit Praxis durch eine Aktion zu verbinden, die auf die Transformation eines gegebenen Kontextes, dh einer bestimmten Realität, abzielt (MIRANDA; RESENDE, 2006). Die Aktionsforschung ermöglicht es also, dem Forschungsprozess die Möglichkeit des Lernens zuzuordnen, da sie die kreative und bewusste Beteiligung sowohl des Forschers als auch der anderen Mitglieder der Forschung erfordert (ROLIM et al., 2004). 
In diesem Zusammenhang zeigt sich die Bedeutung der Aktionsforschung als multidisziplinäres Instrument, da sie gleichzeitig in mehreren Bereichen tätig ist, wie unter anderem im Gesundheitssektor, im Bildungsbereich, in den von Innovation und Wandel geprägten Umgebungen (TRIPP, 2005). Verschiedene Gruppen können betrachtet werden, wie Fachleute, Manager, Studenten und die allgemeine Bevölkerung, sowohl in Gemeinschaften als auch in Institutionen (ROLIM et al., 2004). Es gibt einige Merkmale, die die Aktionsforschung abgrenzen, wie die Konzeptualisierung der Probleme, die bearbeitet werden, die Planung, Ausführung und Bewertung von Maßnahmen zu ihrer Lösung. Es gibt also die Wiederholung dieses Zyklus von Aktivitäten (ANDRÉ, 2000). Neben seinem berüchtigten sozialen Beitrag galt Lewins Arbeit zur Aktionsforschung als innovativ, da sie gleichzeitig den partizipativen und demokratischen Charakter fördert, da sich die Forschung aus der Beteiligung der untersuchten Probanden entwickelt (TRIPP, 2005).

Die Aktionsforschung wird bei ihrer Konstruktion die folgenden Prozesse anwenden: 
Tabelle 1 - Schritte der Aktionsforschung.

\section{ETAPAS DA PESQUISA AÇÃO}

\section{PESQUISA}

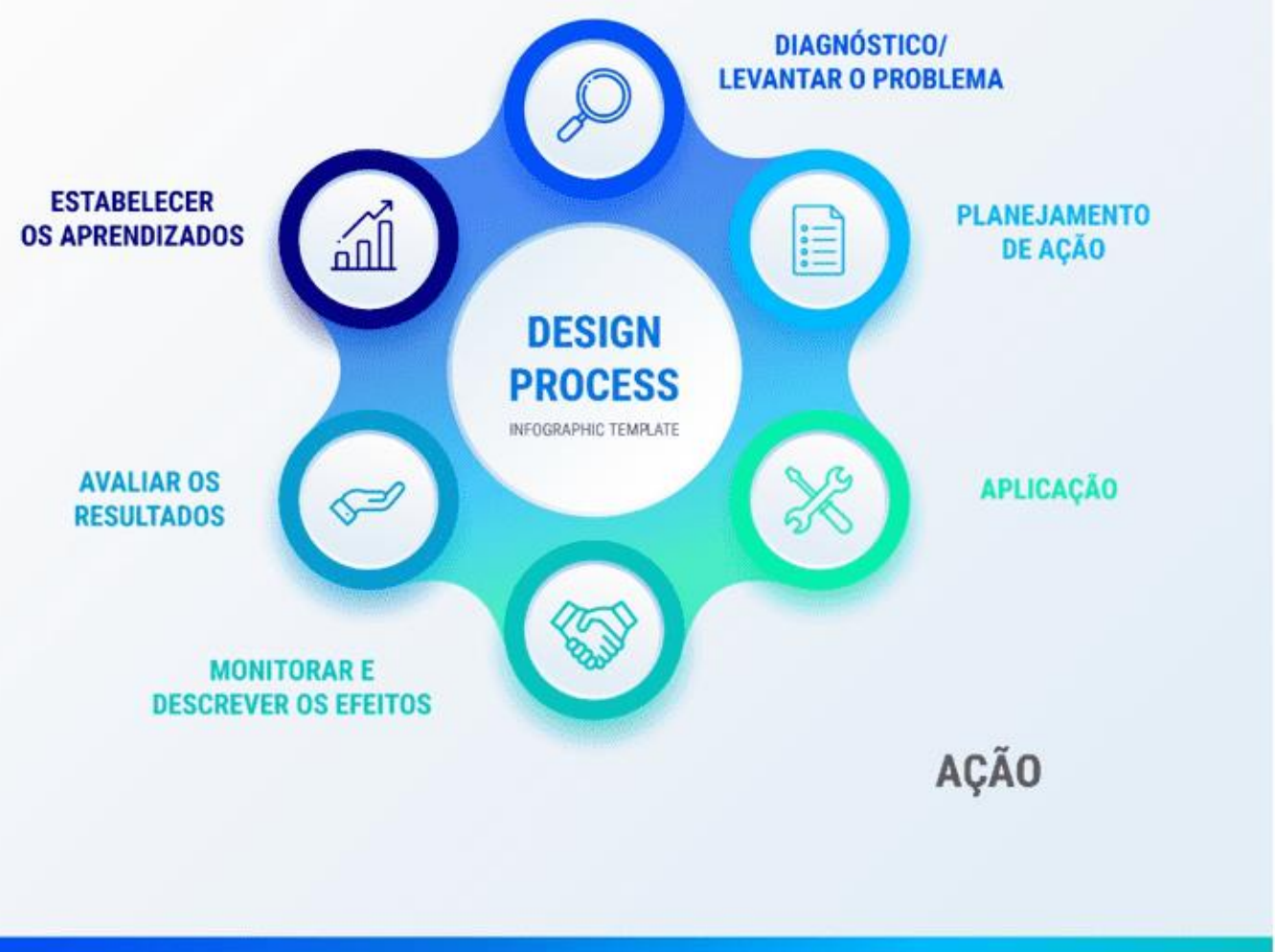

Quelle: Autor.

Daher gibt es eine Studie, die darauf abzielt, Probleme zu lösen, die eine bestimmte Umgebung betreffen, und sich daher auf die Besonderheiten dieses Kontextes konzentriert, in dem sich diese Probleme manifestieren (PEREIRA, 2001; LEWIN, 1946). Ein weiteres Merkmal dieser Art von Forschung ist die Sorge um die wissenschaftliche Gültigkeit der von den Mitarbeitern erhaltenen Ergebnisse. In diesem Prozess werden Diagnosen berücksichtigt, da sie auf die Situation vor und nach dem Einsatz bestimmter Aktionen hinweisen und die detaillierte Aufzeichnung

RC: 102352

Zugangslink: https://www.nucleodoconhecimento.com.br/bildung-de/wissenschaftliche$\underline{\text { methode }}$ 
aller Ereignisse ermöglichen (LEWIN, 1946). Das hier diskutierte Verständnis des Forschungsinstruments bezieht sich auf zwei grundlegende Konzepte, nämlich den Akt der Untersuchung und den materiellen Akt. Der Akt der Untersuchung ist eine Handlung, die in der Lage ist, eine Untersuchung zu verstärken und zu fördern (TRIPP, 2005). Der Substantivakt wiederum ist eine Handlung, die in der Lage ist, eine gewünschte Veränderung in einem bestimmten Kontext zu fördern, der beobachtet und untersucht wird.

Es ist daher ein Prozess, der Forschung mit Handeln verbindet (KOERICH et al., 2009). In diesem Sinne werden die Handlungen in der Klageuntersuchung notwendigerweise als materiell charakterisiert. Aufgrund dieser Qualifikation setzt der Akt der Untersuchung daher eine Verpflichtung voraus, die darin besteht, Menschen zu nützen, die nicht zur wissenschaftlichen Gemeinschaft gehören, aber von den Ergebnissen einer wissenschaftlichen Studie profitieren können (LEWIN, 1946). So übernimmt die Wissenschaft von ihren Forschern eine neue Mission: das an Universitäten produzierte Wissen, ob national oder ausländisch, in die säkulare Gesellschaft zu bringen, so dass die Subjekte, die die unterschiedlichsten sozialen Gruppen ausmachen, emanzipiert werden (FRANCO, 2005). Die von Lewin konzipierte Aktionsforschung wurde auf unterschiedliche Weise und mit unterschiedlichen Zwecken eingesetzt, wodurch ein breites Mosaik theoretischmethodischer Ansätze entstand.

Es gibt mindestens drei verschiedene Konzepte, die in Brasilien auf die Aktionsforschung angewendet werden. Sie werden anhand einiger Kriterien klassifiziert. Der erste Typ ist die kollaborative Aktionsforschung (Transformation wird von der Referenzgruppe an das Forschungsteam angefordert) (KOERICH et al., 2009). Die Rolle des Forschers in diesem Prozess besteht darin, Teil einer Veränderung zu sein, die wissenschaftlich gemacht wird. Dies wird durch die Probanden ausgelöst, die Teil der Gruppe sind (TRIPP, 2005). Es gibt auch critical action research. Die Notwendigkeit einer Transformation wird aus den ersten Studien des Forschers mit der Gruppe (FRANCO, 2005) erkannt. Wenn Veränderung aus einem Prozess resultiert, der die kognitive Konstruktion von Erfahrung schätzt, RC: 102352

Zugangslink: https://www.nucleodoconhecimento.com.br/bildung-de/wissenschaftliche$\underline{\text { methode }}$ 
unterstützt durch kollektive kritische Reflexion, um die Subjekte zu emanzipieren und Bedingungen zu beseitigen, die das Kollektiv als unterdrückend betrachtet, nimmt die Forschung einen wesentlich kritischen Charakter an (ENGEL, 2000). Es gibt auch strategische Aktionsforschung.

Wenn in den anderen Ansätzen die Transformation vorher geplant ist, ohne das aktive Handeln der Probanden, geschieht das gleiche hier nicht. Wenn es nur einen Forscher gibt, der für die Bewertung und Überwachung der Ergebnisse der Anwendung der Studie verantwortlich ist, verliert die handlungskritische Forschung ihre kritische Voreingenommenheit (FRANCO, 2005). Um kritische Aktionsforschung auf diese Weise charakterisieren zu können, bedarf es eines Einbruchs in die Praxis der zu analysierenden sozialen Gruppe, deren Ziel es ist, die latenten Perspektiven zu extrahieren, die die Praktiken unterstützen, d.h. die Veränderungen werden im Kollektiv verhandelt und verwaltet (KOERICH et al., 2009). Auch die kollaborative Aktionsforschung nimmt in diesem Zusammenhang einen kritischen Charakter an (THIOLLENT, 1984). Die Kritikalität, die von dieser Art von Studie ausgeht, erfordert einen Prozess der kollektiven Reflexion über die zu übernehmenden operativen Strategien. Dieser Prozess berücksichtigt sowohl die Stimme des Subjekts als auch seine Perspektiven. Das Interesse nicht nur an der Aufzeichnung und an einer späteren Interpretation.

Sie ist ein untrennbarer Bestandteil dieser Untersuchungsmethodik, so dass sie nicht durch lineare Schritte, die auf die Methode selbst abzielen, etabliert werden kann, sondern aus Situationen und Fakten organisiert ist, die dabei entstehen und wesentlich werden (ENGEL, 2000). Die Betonung des prägenden Charakters dieser Art von Forschung zeigt sich, so dass sich das Subjekt der Transformationen bewusst werden sollte, die sowohl den Forscher als auch die Gruppe und den Prozess betreffen (TRIPP, 2005). Aufgrund dieser Zielsetzung nimmt die Aktionsforschung einen emanzipatorischen Charakter an, so dass sich die an der Forschung beteiligten Subjekte von Mythen und Vorurteilen befreien können, die die Ankunft der gewünschten Veränderungen verhindern (BARBIER, 2002). Diese Art der Forschung wurde im Laufe der Jahre, wie bereits erwähnt, von positivistischen Strömungen RC: 102352

Zugangslink: https://www.nucleodoconhecimento.com.br/bildung-de/wissenschaftlichemethode 
beeinflusst, da die Dialektik der sozialen Realität in den Altag einbezogen wurde, ebenso wie die Punkte, die die Verbreitung einer linearen kritischen Rationalität untermauern und aufrechterhalten.

In diesem Szenario begann der erkenntnistheoretische Status, der die Aktionsforschung unterstützt, sich um die Prozesse hinter der sozialen Transformation zu kümmern, sich den ethischen und politischen Aspekten im Zusammenhang mit der Emanzipation der Subjekte zu widmen und sich auch auf die Bedingungen zu konzentrieren, die den emanzipatorischen Prozess unterstützen (MIRANDA; RESENDE, 2006). Die Aktionsforschung begann, einige interpretative Analyseansätze zuzulassen und erhielt eine Struktur, die in der Lage war, die kritische Beteiligung der Beteiligten zu fördern, was dazu führte, dass der Forschungsprozess die Rekonstruktionen sowie die Resignifikationen von Bedeutungen und Pfaden während der gesamten Ausführung der Phasen zuließ (TRIPP, 2005). Es nahm eine Form an, die als pädagogisch und politisch charakterisiert wurde (THIOLLENT, 1984). Im Kontext der qualitativen Forschung gibt es drei Dimensionen, die von der Aktionsforschung berücksichtigt werden sollten. Die erste davon ist ontologisch. Es geht um die Art des Objekts, das vom Forscher bekannt und untersucht werden soll.

Die zweite Dimension ist erkenntnistheoretisch und betrifft das Subjekt, das es wissen will. Die dritte Dimension schließlich ist die methodische Dimension, deren Ziel es ist, die Prozesse zu kennen, aus denen das Wissen von dem Forscher konstruiert wurde, der an einer bestimmten Gruppe beteiligt ist (FRANCO, 2005). In Bezug auf die ontologische Dimension kann erwähnt werden, dass sie mit einem Leitwissen verbunden ist, und dies sollte es den Subjekten ermöglichen, Wissen für ein besseres Verständnis der Elemente zu produzieren, die eine bestimmte soziale Praxis bedingen (KOERICH et al., 2009). Die Zwecke dieser Forschung werden vom Kollektiv selbst gewünscht, das vom Forscher die Notwendigkeit verlangt, Wissen zu produzieren, das die Umstrukturierung bestimmter Bildungsprozesse ermöglichen kann (FRANCO, 2005). In Bezug auf die Dimension des erkenntnistheoretischen Charakters wird erwähnt, dass der Forscher für seine Ausübung in die Intersubjektivität des Dialekticof des Kollektivs eintaucht.

RC: 102352

Zugangslink: https://www.nucleodoconhecimento.com.br/bildung-de/wissenschaftlichemethode 
Daher muss der Forscher eine differenzierte Haltung im Umgang mit und bei der Interpretation von Wissen einnehmen, da er gleichzeitig versucht, die Realität der untersuchten Gruppe zu kennen und in sie einzugreifen (ENGEL, 2000). Die Vereinigung von Forschung und Handeln führt daher dazu, dass der Forscher im Wesentlichen in das erforschte Universum eintreten muss, aber diese Notwendigkeit macht in keiner Weise die Möglichkeit zunichte, im Umgang mit den Daten eine neutrale Haltung einzunehmen, da dies eine Annahme der Wissenschaft selbst ist (KOERICH et al., 2009). Neutralität ist eine Möglichkeit, die Umstände zu kontrollieren, die eine Forschung durchdringen, was bedeutet, die Aufmerksamkeit auf die grundlegenden erkenntnistheoretischen Annahmen zu lenken, wie die Priorisierung der Dialektik der sozialen Realität und die Historizität von Phänomenen, Praxis, Widersprüchen, Beziehungen zur Totalität und Analyse der Handlung der Subjekte auf der Grundlage einiger Umstände (KOERICH et al. 2009).

Praxis sollte in diesem Zusammenhang als ein grundlegender Weg der Wissenskonstruktion verstanden werden, da durch sie Theorie und Praxis artikuliert werden (FRANCO, 2005). In Bezug auf das zu analysierende Wissen beschränkt sich dies nicht auf die bloße Beschreibung, denn es ist Sache des Forschers, solche Transformationen aus dem zu erklären, was er beobachtet, dh durch eine Bewegung, die die Dialektik des Denkens und Handelns integriert (TRIPP, 2005). Infolgedessen ist das aus dieser Beziehung abgeleitete Wissen in der Lage, die Subjekte und die Umstände, die mit dem Medium zusammenhängen, zu dem es gehört, zu transformieren. Die methodische Dimension wiederum erfordert bestimmte Verfahren, die die Ontologie mit der Epistemologie der Aktionsforschung artikulieren, was bedeutet, in der beobachteten Gruppe eine Dynamik zu etablieren, die die dialogischen, partizipativen und transformativen Prinzipien und Praktiken integriert (TRIPP, 2005). In dieser Dimension müssen einige Elemente berücksichtigt werden.

Unter ihnen kann man die Praxis erwähnen. Es ist der Start- und Ankunftspunkt, wenn es um den Aufbau und/oder die Abmeldung von Wissen geht (FRANCO, 2005). Es basiert auf der Vorstellung, dass Wissen nur durch multiple Artikulationen mit Intersubjektivität konstruiert werden kann. Angesichts dieser Eigenschaft muss RC: 102352

Zugangslink: https://www.nucleodoconhecimento.com.br/bildung-de/wissenschaftlichemethode 
Aktionsforschung, um gut artikuliert zu sein, in der natürlichen Umgebung der zu erforschenden Realität durchgeführt werden, damit die gesammelten Ergebnisse wahr, sicher und von Qualität sind (ENGEL, 2000). In diesem Prozess gibt es eine gewisse Flexibilität, da das Ziel darin besteht, eine Realität im Veränderungsprozess zu erfassen. Daher sollte die Methodik in der Lage sein, dem Forscher zu ermöglichen, bestimmte Anpassungen vorzunehmen, und der Forscher sollte gemäß der vorläufigen Synthese gehen. Solche Zusammenfassungen werden in der Gruppe selbst erstellt (GRITTEM; MEIER; ZAGONEL, 2008). Es sind diese Elemente, die darauf hinweisen, wie ein bestimmter Kontext beeinflusst wurde.

Die Methode sollte eine kontinuierliche Übung von zyklischen Spiralen beinhalten. Diese Spiralen berücksichtigen einige grundlegende Schritte wie Planung, Aktion, Reflexion, Forschungsakte, Resignation und Neuplanung, letzteres bei Bedarf (KOERICH et al., 2009). In Bezug auf die Aktionsforschung wird auch hervorgehoben, dass Bildung und Handeln während eines Ermittlungsprozesses gleichzeitig stattfinden. In diesem Sinne führen die erkenntnistheoretischen und methodischen Gründe, die die Ermittlungstätigkeit durchdringen, dazu, dass Wissen aktiv produziert wird, weil alle am Prozess Beteiligten zusammenarbeiten müssen (ENGEL, 2000). Die Untersuchung ermöglicht es in diesem Zusammenhang, die Veränderungen in der Realität durch Handeln zu verstehen (TRIPP, 2005). Der Prozess erfordert eine bestimmte Haltung des Forschers.

Er kommt oft mit bestimmten Arten von Fragen, sowie mit einigen spezifischen Anforderungen, wie der Einfügung in die Kultur, die neue Bedeutungen, Bedeutungen, Repräsentationen, Widerstände, Erwartungen und Erfahrungen analysiert (FRANCO, 2005). Die Herausforderung, vor der der Forscher wiederum steht, besteht darin, in einem Prozess des Familienaufbaus eine neue Umgebung zu schaffen. Mit anderen Worten, Veränderung sollte von allen Beteiligten wahrgenommen werden (KOERICH et al., 2009). Der Forscher ist einer der Teilnehmer des Universums, das untersucht wird, jedoch ist die Neutralität der Forschung ein Prinzip, das berücksichtigt werden sollte. Daher können ihre Wahrnehmungen über das analysierte Phänomen nicht ignoriert werden, aber seine Handhabung muss in geeigneter Weise erfolgen. Eines RC: 102352

Zugangslink: https://www.nucleodoconhecimento.com.br/bildung-de/wissenschaftlichemethode 
der Ziele, die durch diese Strategie erreicht werden können, betrifft das Erreichen eines größeren Vertrauens unter den Teilnehmern (KOERICH et al., 2009).

Der Aktions-Forschungszyklus beginnt mit der explorativen Phase. Darin wird die Diagnose über die Realität gestellt und eine Umfrage über den Kontext, die anfänglichen Probleme und mögliche Maßnahmen ausgearbeitet (TRIPP, 2005). Daraus legen Forscher und Teilnehmer die Hauptziele der Forschung fest. Diese sollten mit dem beobachteten Bereich, den Akteuren und den Arten von Maßnahmen verknüpft werden, bei denen sie sich auf die Ermittlungstätigkeit konzentrieren sollen (FRANCO, 2005). Das Thema der Forschung wird nacheinander definiert. Dies wird von einem praktischen Problem im Zusammenhang mit einem Suchgebiet getrennt. Dies wird auf der Grundlage der Verpflichtungen ausgewählt, die zwischen dem Forscherteam und den Themen eingegangen wurden, die der jeweiligen Situation entsprechen (FRANCO, 2005). Das Thema kann aber auch von den Akteuren der Situation angefordert werden. Es sollte sowohl für die Forscher als auch für die untersuchten Probanden von Interesse sein, damit alle eine aktive Rolle spielen und zur Entwicklung der Forschung beitragen.

Es wählt auch einen spezifischen theoretischen Rahmen, mit dem gearbeitet werden soll, weil es die gesamte Forschung leiten wird. Drittens werden die Probleme gewählt, d.h. das Forschungsproblem. Aus dem Problem gewinnt das Thema an Robustheit (ENGEL, 2000). Das Problem sollte einige Forschungsannahmen umfassen, wie die Analyse und Abgrenzung der Ausgangssituation; die Gestaltung der endgültigen Situation auf der Grundlage der Kriterien der Behinderung und der Durchführbarkeit; die Identifizierung aller zu lösenden Probleme; die Umsetzung dieser Probleme in entsprechende Maßnahmen; und schließlich die Umsetzung und Bewertung von Maßnahmen (TRIPP, 2005). In diesem Sinne ist es notwendig, einen Rahmen theoretischer Bezüge an die praktische Realität der Forschung anzupassen. Das Ziel ist, dass die Informationen von einer theoretischen Basis analysiert und interpretiert werden, jedoch müssen sie mit den realen Erfahrungen artikuliert werden, die daher von den Mitarbeitern der Studie gesammelt wurden (TRIPP, 2005). 
Sie kommen zu den Quoten. Trotz der falschen Vorstellung, dass es keine Hypothesen gibt, ist es notwendig, sorgfältig über diese Aussage nachzudenken, was impliziert, die Hypothesen als vom Forscher formulierte Annahmen zu verstehen, die bestätigt werden können oder nicht (KOERICH et al., 2009). Die Hypothesen lassen mögliche Lösungen für ein Forschungsproblem $\mathrm{zu}$ und zielen somit darauf $\mathrm{ab}$, einen Gedankengang zu führen (KOERICH et al., 2009). Das Seminar ist auch eine der Phasen der Aktionsforschung, da es eine bedeutende Rolle im Entscheidungsprozess in einer Untersuchung spielt und die Koordination von Aktivitäten ermöglicht. Der Zweck eines Seminars besteht darin, das Thema zu definieren und Probleme zu ermitteln, mit denen die Forschung arbeiten wird. Die Ausarbeitung des Problems, so dass Probleme behandelt werden, sowie Forschungshypothesen, ist eines der Ziele. Die Studiengruppen und Forschungsteams, die diese Aktivitäten koordinieren, sind geboren.

Die Informationen stammen aus verschiedenen Quellen und Gruppen, was den Zugang zu Interpretationen ermöglicht, die die Prozesse der Erstellung von Aktionsleitlinien fördern, sowie es ist möglich, die Maßnahmen zu bewerten und die Ergebnisse aus geeigneten Kanälen zu verbreiten (KOERICH et al., 2009). Auch die Aktionsforschung wird aus den Bereichen Beobachtung, Stichprobenziehung und qualitative Repräsentativität operationalisiert. Dies kann eine konzentrierte und/oder verteilte Gemeinschaft umfassen, jedoch ist Stichprobenbildung und Repräsentativität ein Faktor, der diskutiert werden kann. Einige Studien schließen die Stichprobe aus, andere empfehlen ihre Verwendung, andere bewerten die Kriterien der qualitativen Repräsentativität (FRANCO, 2005). Sie gelangen in die Datenerfassungsphase. Die wichtigsten verwendeten Techniken sind kollektive und/oder individuelle Interviews, konventionelle Fragebögen, das Studium von Akten usw. Nach der Sammlung von Informationen aus den Beobachtungsgruppen werden diese gemeinsam diskutiert, analysiert und interpretiert. 


\section{ENDGÜLTIGE ÜBERLEGUNGEN}

Der Prozess der Ermittlungstätigkeit, der Realitätsanalyse sowie der Bewertung qualitativer Werkzeuge und Methoden erfordert eine gewisse Dialogizität, dh er impliziert die Suche nach reflektierendem Wissen und die Festigung eines Engagements für die konkrete Realität. Mit anderen Worten, es erfordert eine effektive Anerkennung des zu analysierenden Themas und der zu analysierenden Realität durch eine dynamische Bewegung zwischen den an der Untersuchung beteiligten Parteien. Diese zirkuläre und dynamische Möglichkeit liegt in der Resonanz und Unterstützung für die Eroberung eines neuen Raumes und/oder die Eroberung eines neuen Wissens. So analysiert die Aktionsforschung als methodisches Werkzeug menschliches Handeln aus einer kommunikativen und partizipativen Bewegung, die den Austausch von Wissen und die Ausarbeitung einer relationalen Struktur des Vertrauens und des Engagements für die zu transformierenden Subjekte begünstigt. Ihr Ziel ist die Lösung oder Klärung der in der Gruppe auftretenden Probleme.

Während des Prozesses besteht die Notwendigkeit einer kontinuierlichen Überwachung von Entscheidungen, Handlungen und allen absichtlichen Aktivitäten, die von den an der Situation beteiligten Akteuren entwickelt wurden. Daher ist die Forschung nach diesem Ansatz nicht nur auf eine Aktionsform beschränkt, da sie darauf abzielt, das Wissen der Forscher sowie ihren Bekanntheitsgrad über die beteiligten Personen und / oder Gruppen zu erhöhen. Die Forschung fördert viel mehr als eine Datenerhebung und/oder Vorschläge praktischer Interventionen. Neben den hier vorgestellten Überlegungen wird betont, dass die Handlungsforschung unter anderem reale, aktuelle und kohärente Diskussionen und Erklärungen aggregiert, die die Generierung von deskriptivem, wenn auch kritischem Wissen über die in verschiedenen sozialen Umfeldern erlebten Situationen ermöglichen. Es fördert daher neue Formen des Ausdrucks und der Reflexion über die Bedeutungen und Gefühle der Teilnehmer, die bei der Analyse der jeweiligen Problemsituation zugeschrieben werden. 


\section{VERWEISE}

ANDRÉ, M. E. D. A. Etnografia da prática escolar. Série Prática Pedagógica. Campinas: Papirus; 2000.

BARBIER, R. A pesquisa-ação. Tradução Lucie Didio. Brasília: Plano, 2002.

CARR, W.; KEMMIS, S. Teoria crítica de la enseñanza. Barcelona: Martinez Roca, 1988.

ENGEL, G. I. Pesquisa-ação. Educar em Revista, n. 16, p. 181-191, 2000.

FRANCO, M. A. S. Pedagogia da pesquisa-ação. Educação e pesquisa, v. 31, n. 3, p. 483-502, 2005.

GRITTEM, L.; MEIER, M. J.; ZAGONEL, I. P. S. Pesquisa-ação: uma alternativa metodológica para pesquisa em enfermagem. Texto \& Contexto-Enfermagem, v. 17, n. 4, p. 765-770, 2008.

$\mathrm{KOERICH}, \mathrm{M}$. S. et al. Pesquisa-ação: ferramenta metodológica para a pesquisa qualitativa. Revista Eletrônica de Enfermagem, v. 11, n. 3, p. 717-723, 2009.

LEWIN, K. Problemas de dinâmica de grupo. São Paulo: Cultrix, 1946.

MIRANDA, M. G. de.; RESENDE, A. C. A. Sobre a pesquisa-ação na educação e as armadilhas do praticismo. Revista Brasileira de Educação, v. 11, n. 33, p. 511-518, 2006.

MORIN, A. Pesquisa-ação integral e sistêmica: uma antropopedagogia renovada. Tradução Michel Thiollent. Rio de Janeiro: DP\&A, 2004.

PEREIRA, E. M. A. Professor como pesquisador: o enfoque da pesquisa-ação na prática docente. In: GERALDI, C. M. G.; FIORENTINI, D.; PEREIRA, E. M. A. (Org). Cartografias do trabalho docente: professor(a) - pesquisador(a). Coleção Leituras no Brasil. Campinas: Mercado das Letras, 2001. p. 153-81.

RC: 102352

Zugangslink: https://www.nucleodoconhecimento.com.br/bildung-de/wissenschaftlichemethode 
ROLIM, K. M. C. et al. Mulheres em uma aula de hidroginástica: experenciando o interrelacionamento grupal. Revista Brasileira em Promoção da Saúde, v. 17, n. 1, p. 8-13, 2004.

THIOLLENT, M. Notas para o debate sobre pesquisa-ação. In: BRANDÃ O, C. (Org.). Repensando a pesquisa participante. São Paulo: Brasiliense, 1984. p. 82-103.

TRIPP, D. Pesquisa-ação: uma introdução metodológica. Educação e pesquisa, v. 31, n. 3, p. 443-466, 2005.

Eingereicht: November 2021.

Genehmigt: November 2021. 